\title{
LETTER TO THE EDITOR \\ International spinal cord injury upper extremity basic data set version 1.1
}

\author{
Spinal Cord (2015) 53, 890; doi:10.1038/sc.2015.101; published online 9 June 2015
}

In the International Spinal Cord Injury Upper Extremity Basic Data Set $^{1}$ we have adjusted the wording of a variable's description and a variable's coding that we would like to bring to the attention of your readers.

The following two changes to the International Spinal Cord Injury Upper Extremity Basic Data Set from Version 1.0 to Version 1.1 are:

In relation to the variable 'Basic hand-upper extremity function' the following sentence has been added to the Description of the variable: 'or functional gain after upper extremity reconstructive surgery or during or after the use of a neuroprosthesis' - this will enable the same scoring to be used following these interventions:

VARIABLE NAME: Basic hand-upper extremity function

DESCRIPTION: This variable consists of two items, one for the right upper extremity and one for the left upper extremity. Each item describes the hand-arm function as it relates to motor innervation, or functional gain after upper extremity reconstructive surgery or during or after the use of a neuroprosthesis.

In relation to the variable 'Shoulder function classification', the option 'D. Full range of movement (ROM) of shoulder and independent reaching forward and upward' was changed to 'D. Ability to reach in all directions including lifting hand above the head reflecting at least grade 3 strength in the shoulder flexors and abductors and elbow extensors'. The modification was made because option D was not capturing the same construct as options A-C. Options A-C are descriptions of abilities, whereas option D was solely a description of strength even though it was not explicitly stated. In addition, the new definition now distinguishes between active and passive movement for clarity.

VARIABLE NAME: Shoulder function classification

CODES: Each side is scored separately according to the following 4-point scale: A. No active placing or reaching of the arm.

B. Severely limited but able to position hand on a desk, without assistance, but not able to reach to the mouth/head (gravity compromises the movements).

C. Limited but able to reach mouth/head with difficulty or altered movements, for example, weak or absent pronation-supination or wrist flexion-extension.

D. Ability to reach in all directions including lifting hand above the head reflecting at least grade 3 strength in the shoulder flexors and abductors and elbow extensors.
The adjustments are now found on ISCoS website: http://www. iscos.org.uk/international-sci-upper-extremity-data-sets, together with training cases for Version 1.1 of the International Spinal Cord Injury Upper Extremity Basic Data Set.

\section{CONFLICT OF INTEREST}

The authors declare no conflict of interest.

F Biering-Sørensen ${ }^{1}$, A Bryden ${ }^{2}$, A Curt $^{3}$, J Friden ${ }^{4}$, LA Harvey ${ }^{5}$, MJ Mulcahey ${ }^{6}$, MR Popovic ${ }^{7}$, A Prochazka ${ }^{8}$, KA Sinnott ${ }^{9}$ and $\mathrm{G}$ Snoek ${ }^{10}$

${ }^{1}$ Department for Spinal Cord Injuries, Rigshospitalet and Glostrup Hospital, University of Copenhagen, Copenhagen, Denmark;

${ }^{2}$ The Cleveland FES Center/Case Western Reserve University, Cleveland, $\mathrm{OH}, \mathrm{USA}$;

${ }^{3}$ Spinal Cord Injury Center, University of Zurich and University Hospital Balgrist, Zurich, Switzerland; ${ }^{4}$ Department of Hand Surgery, Institute of Clinicalsciences, Sahlgrenska University Hospital and University of Gothenburg, Gothenburg, Sweden;

${ }^{5}$ Rehabilitation Studies Unit, Northern Clinical School, Sydney School of Medicine, University of Sydney, Sydney, New South Wales, Australia; ${ }^{6}$ Jefferson School of Health Professions, Thomas Jefferson University, Philadelphia, PA, USA;

${ }^{7}$ Institute of Biomaterials and Biomedical Engineering, University of Toronto, Toronto, Ontario, Canada;

${ }^{8}$ Division of Neuroscience, University of Alberta, Edmonton, Alberta, Canada;

${ }^{9}$ Burwood Academy of Independent Living, Burwood Hospital and Department of Orthopaedic Surgery and Musculoskeletal

Medicine, University of Otago, Christchurch, New Zealand and

${ }^{10}$ Roessingh Rehabilitation Center, Enschede, The Netherlands E-mail: fin.biering-soerensen@regionh.dk

1 Biering-Sørensen F, Bryden A, Curt A, Friden J, Harvey LA, Mulcahey MJ et al. International spinal cord injury upper extremity basic data set. Spinal Cord 2014; 52: 652-657. 